\title{
SEMG Signals BP Neural Network Classification Based on Wavelet Packet Energy
}

\author{
Liu-yang XU, Li-juan SHI, Yi YU, Xin-qi HE, Yun ZHAO and \\ Jun-tang LIN
}

Xinxiang Medical University, HeNan, China

Keywords: Surface EMG, Wavelet transform, Wavelet packet, BP neural network.

\begin{abstract}
The purpose of this paper is to study the different features of surface electromyography signals while subjects take different actions with their forearms. In this paper, the surface electromyography signals, recorded from some healthy volunteers under different actions (fist, fist exhibition, inside wrist pronation and wrist supination), were first denoised by wavelet packet transform. And then we extracted the features of the surface electromyography signals. Finally, the BP neural network was utilized to classify the features so as to distinguish different forearm actions. These results can be used as reference data in muscular diseases and disability in patients treatment or diagnosis .In this article, it was the first time to study surface electromyography signals, and this signal was generate by healthy volunteer under different action of fist ,fist exhibition inside wrist pronation and wrist supination. The method of this study could be used in produced intelligent prostheses.
\end{abstract}

\section{Introduction}

Since the 17th century, scientists began to study sEMG [1], From the beginning to confirm muscle contraction is associated with the change of electrical power to the electromyographic signal found muscle contraction, scientists study a step by step the principles an. With the development of science and technology, technical analysis of EMG quickly permeated all muscle-related fields, and gradually become a hot topic of the study.

The study found that muscle stimulation encountered some time, in which the muscle fibers will be excited, and then generate action potentials, there has been a stimulus, it will have the potential sequences. It will define the overall motor unit motor unit action potentials generated sequence of physiological EMG, EMG is [3]. Surface EMG was collected by surface electrodes, this avoiding traumatic defects. And because of this strength, It has been widely applied to check the body movement, muscle diseases diagnosis and other fields. With the development of technology, people study the surface EMG has also been great progress, such as: the development of myoelectric prostheses, functional electrical stimulation, sports medicine [2]. Therefore, the study of surface EMG to our understanding of the human nervous have a significant impact.

\section{Test Method and Content \\ EMG Collection Electrode}

EMG electrodes can be divided into the needle electrode and the surface electrode. Needle electrode is surgically implanted wire method internal to the muscles, such electrodes can be done through targeted acquisition, the acquired EMG more precise, low noise, but easy to produce injury subjects, impact the health of test, often used in 
animal experiments. Surface electrode is an electrode paste to the skin surface of the subject, the muscle cells produce current through the skin to the electrode conductivity. This collection method to collect electrodes harm to subjects is relatively small, used widely. In this paper, using surface electrodes acquisition subject forearm muscle EMG.

\section{EMG Acquisition Position}

Skeletal muscle is an important part of human muscle, skeletal muscle collected at EMG relatively easy to distinguish observed. Human hand is one of the most flexible part of the arm near the EMG collected skeletal muscle has a waveform obvious, easy to operate and so on. Electrode paste discharge position of the forearm flexor carpi ulnaris, flexor carpi at the experimenter in the right arm upward intervals along the fiber direction $2 \mathrm{~cm}$, respectively, From electrical and mechanical principle can follow the electromyographic signal strongest muscle belly ${ }^{[7]}$ according to the text experimental needs, EMG collection area for the flexor carpi, its physiological role is to bend the wrist, inner wrist close. In this part of the collected energy EMG changes obviously, easy to operate, it is in line with the needs of the research topic.

\section{Electromyography Collection Content}

Before collection SEMG, we should wipe the skin by $75 \%$ of medical alcohol to get rid of the skin surface layer of grease and stains, the electrode sheet has good skin contact. During EMG signal acquisition, acquisition experimenters do show fist, make a fist, wrist rotation within, EMG wrist supination action of the four groups, each group collected 6 data movement requirements are as follows:

(1) fist: natural relaxation palm palms up, after $1 \mathrm{~s}$ fist, after holding $2 \mathrm{~s}$, and finally release and restore the initial state

(2) exhibition boxing: the hand was a fist state palms up, palms after 1s after release, holding $2 \mathrm{~s}$, and finally make a fist and restore the initial state

(3) inner wrist rotation: natural relaxation palm palms up, after $1 \mathrm{~s}$ after rotating the wrist to the inside of the human body flip, while fist, after $2 \mathrm{~s}$, turn the wrist outward while flipping the body release the palm and restore the initial state

(4) wrist supination: Nature relaxation palm palms down, after $1 \mathrm{~s}$ after rotating the wrist to the outside of the body flip, while fist, after $2 \mathrm{~s}$, turn the wrist inward flip at the same time release the body to restore the initial state and palm

\section{SEMG Feature Extraction and Classification}

\section{Surface and Noise Cancellation}

SEMG is a very weak signal, the amplitude generally in the range of $100 \sim 5000 \mu \mathrm{V}$, peak -peak amplitude between $0 \sim 6 \mathrm{mV}$, the desired signal frequency is in the range of $0 \sim 500 \mathrm{~Hz}$, mainly in the energy range of $50 \sim 150 \mathrm{~Hz}^{[5]}$, the surface EMG which collected by surface electrodes contains a lot of noise (such as heart sound noise, frequency interference, friction between the electrode and the skin and so on).In measuring the forearm flexor wrist movement in different through multi-channel semg digital band-pass filter, bandpass filter of low-frequency cutoff frequency is set to $10 \mathrm{Hertz}$, high-frequency cutoff frequency is set to $500 \mathrm{hz}$, filter out unwanted noise. 


\section{Based on Surface EMG Wavelet Analysis Extract}

Since the Fourier transform is applied, it has been the most effective method for processing signal, but it is not in the time domain resolving power. In order to study the local signal characteristics in time, scientists have proposed short-time Fourier transform, short time Fourier transform also has its limitations, so scientists have developed a wavelet transform, wavelet transform not only the development of the short-time Fourier transform localized thought they solved the problem of short-time Fourier transform. It is suitable to deal with similar problems surface EMG signals to mutations. Wavelet analysis is frequency analysis AFP recently a time-out, it is widely used in signal processing problems. This article is on the EMG four finger movement pattern feature vectors extracted by wavelet transform and wavelet packet respectively. The wavelet transform is to use multi-resolution signal decomposition. This article will take a wavelet transform signal decomposition and reconstruction 4 scale signal analysis to extract feature vector signal. Under its decomposition coefficients is a waveform reconstructed surface EMG waveform fist, it is db2 wavelet decomposition layer 4,as show in the figure 1,2, the original signal and reconstructed signal reconstructed approximation detail signal. As can be seen in Figure 3, d3, d4, a4 is the main area of the original signal energy. And do analysis for Fist of the exhibition, in the wrist rotation, external rotation of the wrist surface sEMG shows that they are the main energy region $\mathrm{d} 3, \mathrm{~d} 4, \mathrm{a} 4$, so this press 4 on the scale signal analysis, and finally the use of $\mathrm{d} 1, \mathrm{~d} 2, \mathrm{~d} 3, \mathrm{~d} 4, \mathrm{a} 4$ as part of the classification characteristics of the signal.

In order to analyze the surface EMG better, we make $a=8,16,32$ respectively of its multi-scale decomposition and reconstruction respectively, Figure 2 shows the energy distribution of surface EMG in the low frequency region. Therefore, this article will use $\mathrm{d} 1, \mathrm{~d} 2, \mathrm{~d} 3, \mathrm{~d} 4, \mathrm{a} 4, \mathrm{a} 8, \mathrm{a} 16, \mathrm{a} 32$ as feature vectors.
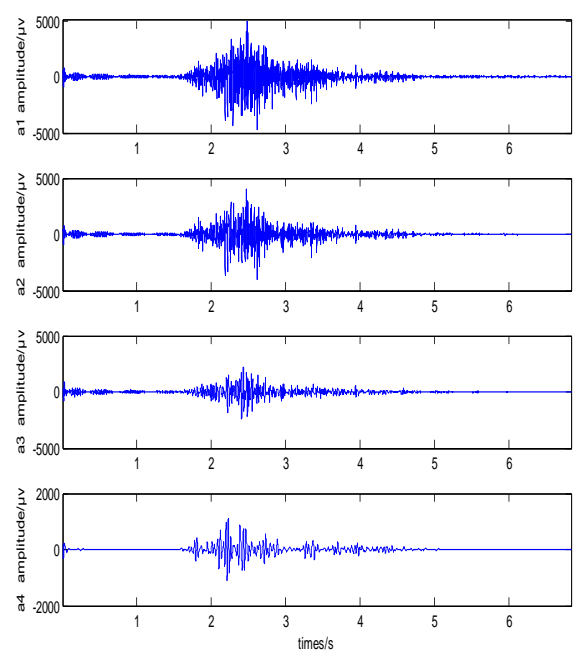
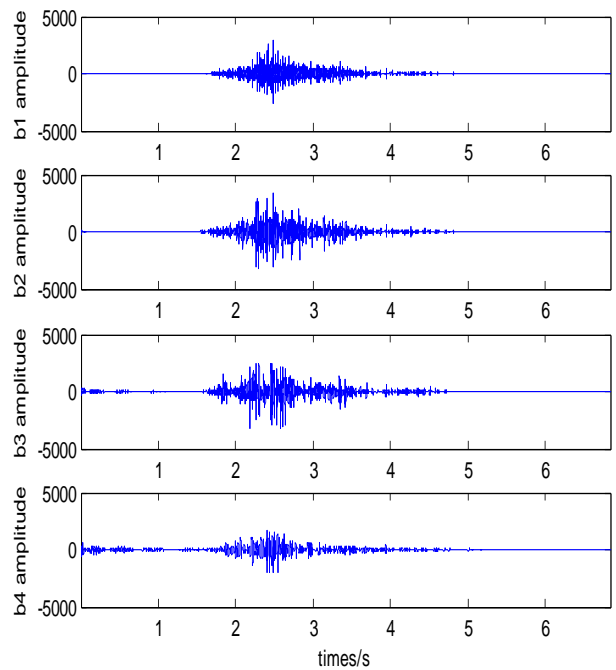

Fig1refactoring 1 4 layer detail signalFig2 refactoring 1 4 layer approximation signal

Let fist maximum signal frequency component is 1 , the decomposed signal levels occupied frequency range as shown in Table 1. 
Table 1 five different sEMG signal energy distribution in wavelet domain

\begin{tabular}{|c|c|c|c|c|c|}
\hline Finger Sprint & $\mathrm{d} 1$ & $\mathrm{~d} 2$ & $\mathrm{~d} 3$ & $\mathrm{~d} 4$ & $\mathrm{a} 4$ \\
\hline normal & & & & & \\
\hline Make a fist & 3.64 & 10.79 & 20.94 & 13.05 & 51.56 \\
\hline Spread figure & 3.44 & 10.75 & 21.76 & 12.34 & 51.69 \\
\hline Wrist inward turning & 6.64 & 15.62 & 18.94 & 10.48 & 48.31 \\
\hline Wrist outer turning & 8.58 & 17.44 & 17.29 & 9.48 & 47.21 \\
\hline
\end{tabular}

\section{SEMG Based on Wavelet Packet Extraction}

EMG is a very weak signal, wavelet packet transform could improve the time resolution of the signal, which display a more detailed spectral information, and accurately express the EMG information. This artical Put forward to using wavelet packet transform analysis of signals of different finger movement patterns extracted feature vectors. SEMG signals fist set maximum energy component is 1, the decomposition of wavelet packet signal levels relative share of wavelet packet energy range as shown in table 2

Table2 SEMG signals relative wavelet energy distribution

\begin{tabular}{|c|c|c|c|c|c|c|c|c|}
\hline P1-P8 & P1 & P2 & P3 & P4 & P5 & P6 & P7 & P8 \\
\hline Percent(\%) & 12.23 & 23.66 & 16.42 & 21.52 & 2.06 & 2.55 & 10.27 & 4.68 \\
\hline P9-P16 & P9 & P10 & P11 & P12 & P13 & P14 & P15 & P16 \\
\hline Percent(\%) & 0.20 & 0.29 & 0.59 & 0.40 & 1.99 & 1.28 & 0.69 & 1.15 \\
\hline
\end{tabular}

We can see in the table2 that the signal main energy area focused on the decomposition tree node, and we also could get this conclusion by other modle signal. So take these nodes as the relative wavelet packet energy eigenvector

\section{Neural Network Classification Based on SEMG Signal Feature Vectors}

Construction of BP Neural Network. In this article, we discuss four finger movement pattern signal, the data collected from inside the extracted feature value divided Two groups, one for the neural network classifier training set, and the other used to examine the classification accuracy. If the output point value $\geq 0.5$, and the remaining nodes are $\leq 0.5$ output signal is said to be recognized as a category. According to the characteristics and requirements of input output to determine the input layer and output layer, the number of hidden layer neurons, general input function approximation, the hidden layer neurons, the more the more accurate approximation, classification and the more accurate. Which BP network has been designed, then the part is the realization of BP network: the permissible error setting, step length setting and set the initial weights and bias. System to the training goal is to allow error, this paper set the margin of error is $0.001 \sim 0.001$,;The BP neural network design is the key choice of learning step, learning step basic set in $0.02 \sim 0.2$; When the initial weights and bias shoulds not be too great attention should be paid to the initial weights and bias, the weight can not set the same value, this paper mainly use random number $(1,1)$ enactment right values and offset. 
BP Neural Network Classification Based on Wavelet Packet Energy. Spend sections designed BP neural network classifier, input composition was the Wavelet Packet Energy Eigenvector $\left(P_{4}^{1}, P_{4}^{2}, P_{4}^{3}, P_{4}^{4}, P_{4}^{5}, P_{4}^{6}, P_{4}^{7}, P_{4}^{9}, P_{4}^{11}, P_{4}^{13}\right)$ that by obtained above, the output nodes 10 ,named the four sports mode was output, expressed in terms of a finger without moving [0 000], fist to [1000], show boxing is [0100] in the wrist spin of [0010], wrist supination [0001], the number of nodes is 4, and Like the previous section, the hidden layer nodes for classification 4 , the training and testing

Table 3 Feature Extraction Based on Wavelet Packet classification results

\begin{tabular}{|l|c|c|c|c|c|}
\hline & $\begin{array}{c}\text { make a } \\
\text { fist }\end{array}$ & Fist Show & $\begin{array}{l}\text { Inner wrist } \\
\text { rotation }\end{array}$ & $\begin{array}{l}\text { Wrist } \\
\text { supination }\end{array}$ & $\begin{array}{l}\text { Stationary } \\
\text { finger }\end{array}$ \\
\hline $\begin{array}{l}\text { Number of } \\
\text { training samples }\end{array}$ & $30 / 30$ & $30 / 30$ & $30 / 30$ & $30 / 30$ & $30 / 30$ \\
\hline $\begin{array}{l}\text { Number of } \\
\text { training samples }\end{array}$ & $30 / 29$ & $30 / 28$ & $30 / 27$ & $30 / 28$ & $30 / 30$ \\
\hline $\begin{array}{l}\text { Number of } \\
\text { training samples }\end{array}$ & $96.7 \%$ & $93.3 \%$ & $90.0 \%$ & $93.3 \%$ & $100 \%$ \\
\hline
\end{tabular}

We can see from the table that the basic classification results more than $90 \%$, better classification results.

Fingers in the human activity in a very important role, and now available for the finger surface electromyography studies have not reached a common level, this paper research for surface EMG of different finger movements, through the acquisition of the signal waveform to dry processing, the results from our process can be seen mainly in the frequency $0 \sim 500 \mathrm{~Hz}$, energy mainly between $50 \sim 100 \mathrm{~Hz}$, for the results of the study, we can work out the range of finger motion by a range of frequencies and energy, and wavelet transform and wavelet packet signal feature extraction with 4 scale decomposition feature vector signal, we know that its main energy coverage within $\mathrm{d} 3, \mathrm{~d} 4$, a4. Finally, its classification, classification result more than $90 \%$, better classification results.

\section{Conclusion}

This article describes the opponent forearm flexor carpi in hand to do the surface EMG signal acquisition fist, fist exhibition, inside wrist pronation, wrist external rotation and other movements, to do sEMG wavelet transform, de-noising, feature extraction and other analysis. According to the characteristics of EMG ,we do the frequency filtering, eliminate noise SEMG. After filtering, WE can discovery that the amplitude of the surface of the electrical signal is significantly smaller. Through its power spectrum found that higher energy situation at a certain frequency of occurrence of the disappearance of the energy surface electromyography smoother distributed within the range of $0 \sim 600 \mathrm{HZ}$. And we also using wavelet transform signal decomposition and reconstruction 4 scale signal analysis to extract feature vector signal, based on the analysis of EMG done a deal with BP neural network classification, classification result more than $90 \%$ better classification results. This indicates that the above described method of surface EMG signal processing can be used to treat orthopedic, muscle type diseases. 


\section{References}

[1] Tianpei Hu. study found that EMG and EMG Rehabilitation [J].in chinese Shanghai Jiaotong University, 1994,28 (3);.. 151-153.

[2] Liyu Cai,Zhong-zhi Wang,and Hai-hong Zhang ,EMG Signal Classification Method Based on Wavelet Transform.in chinese Data Acquisition pretreatment, [J]2000, 158 (2): 255-258.

[3] Lei Liu, Wenhao. Yueneuromuscular FIG principle .in chinese Beijing;Science and Technology Press, 1983.

[4] $\mathrm{Mu}$ Guizhen common principles and use of electronic medical equipment.in chinese Beijing; Electronic Industry Press, 1995.

[5] (German) Karl et al., And Color Atlas of Human Anatomy, Jinan;in chinese Shandong Science and Technology Press, 2000: 136-148.

[6] LeiLu, etc. the limb Experimental Study of based on Surface EMG to distinguish upper movements. in chinese. Master's thesis, 2011. 